\title{
Decompositions for real Banach spaces with small spaces of operators
}

\author{
by \\ Manuel GonzÁlez and José M. Herrera (Santander)
}

\begin{abstract}
We consider real Banach spaces $X$ for which the quotient algebra $\mathcal{L}(X) / \mathcal{I} n(X)$ is finite-dimensional, where $\mathcal{I} n(X)$ stands for the ideal of inessential operators on $X$. We show that these spaces admit a decomposition as a finite direct sum of indecomposable subspaces $X_{i}$ for which $\mathcal{L}\left(X_{i}\right) / \mathcal{I} n\left(X_{i}\right)$ is isomorphic as a real algebra to either the real numbers $\mathbb{R}$, the complex numbers $\mathbb{C}$, or the quaternion numbers $\mathbb{H}$. Moreover, the set of subspaces $X_{i}$ can be divided into subsets in such a way that if $X_{i}$ and $X_{j}$ are in different subsets, then $\mathcal{L}\left(X_{i}, X_{j}\right)=\mathcal{I} n\left(X_{i}, X_{j}\right)$; and if they are in the same subset, then $X_{i}$ and $X_{j}$ are isomorphic, up to a finite-dimensional subspace. Moreover, denoting by $\widehat{X}$ the complexification of $X$, we show that $\mathcal{L}(X) / \mathcal{I} n(X)$ and $\mathcal{L}(\widehat{X}) / \mathcal{I} n(\widehat{X})$ have the same dimension.
\end{abstract}

1. Introduction. Gowers and Maurey obtained in [12] the first example of an infinite-dimensional Banach space $X_{\mathrm{GM}}$ which is hereditarily indecomposable, in the sense that no subspace of $X_{\mathrm{GM}}$ can be written as the direct sum of two infinite-dimensional closed subspaces. This space provided a negative answer to several long-standing open problems in Banach space theory. Moreover, both in the real case $\mathbb{F}=\mathbb{R}$ and in the complex case $\mathbb{F}=\mathbb{C}$, every operator $T$ acting on $X_{\mathrm{GM}}$ can be written as $T=\lambda I+S$, with $\lambda \in \mathbb{F}$ a scalar number and $S \in \mathcal{S S}\left(X_{\mathrm{GM}}\right)$, where $\mathcal{S S}(X)$ denotes the ideal of strictly singular operators acting on the Banach space $X$. Afterwards, many examples of hereditarily indecomposable spaces and some other classes of spaces with unexpected properties were constructed. We refer to [17] for a readable paper describing some basic constructions and interesting examples of spaces of this kind. Moreover, [10] is a short survey describing the relation between these spaces and Fredholm theory, as well as many examples of spaces $X$ for which $\mathcal{L}(X) / \mathcal{S} \mathcal{S}(X)$ is small.

2000 Mathematics Subject Classification: Primary 47A53.

Key words and phrases: real hereditarily indecomposable Banach spaces, complexification, Fredholm ideals.

Supported in part by DGI (Spain), Grant MTM2007-67994. 
Let $X$ be an infinite-dimensional, hereditarily indecomposable Banach space. It is not difficult to see that in the case $\mathbb{F}=\mathbb{C}$, the quotient algebra $\mathcal{L}(X) / \mathcal{S} \mathcal{S}(X)$ is one-dimensional, i.e., can be identified with $\mathbb{C}$. However, the case $\mathbb{F}=\mathbb{R}$ is more complicated. It was proved by Ferenczi $[5,6]$ that in the real case $\mathcal{L}(X) / \mathcal{S S}(X)$ is isomorphic as a real algebra to either the real numbers $\mathbb{R}$, the complex numbers $\mathbb{C}$, or the quaternion numbers $\mathbb{H}$. Recently, he showed in [7] that there are two examples of real hereditarily indecomposable spaces for which $\mathcal{L}(X) / \mathcal{S S}(X)$ is $\mathbb{C}$ and $\mathbb{H}$, respectively. So we have examples for the three possible cases.

In this paper, we consider real Banach spaces $X$ for which $\mathcal{L}(X) / \mathcal{I} n(X)$ is finite-dimensional, where $\mathcal{I} n(X)$ stands for the inessential operators on $X$. We show that these spaces $X$ admit a decomposition

$$
X=\bigoplus_{i=1}^{k}\left(\bigoplus_{j=1}^{n_{i}} Y_{i j}\right)
$$

as a direct sum of indecomposable subspaces $Y_{i j}, 1 \leq i \leq k, 1 \leq j \leq n_{i}$, where $\mathcal{L}\left(Y_{i j}\right) / \mathcal{I} n\left(Y_{i j}\right)$ is isomorphic as a real algebra to $\mathbb{R}, \mathbb{C}$, or $\mathbb{H}$. We also show that, given two of these subspaces $Y_{i j}$ and $Y_{i^{\prime} j^{\prime}}$, every operator in $\mathcal{L}\left(Y_{i j}, Y_{i^{\prime} j^{\prime}}\right)$ is inessential when $i \neq i^{\prime}$, and there exists a Fredholm operator from $Y_{i j}$ to $Y_{i^{\prime} j^{\prime}}$ when $i=i^{\prime}$. Moreover, denoting by $\widehat{X}$ the complexification of $X$, we show that $\mathcal{L}(\widehat{X}) / \mathcal{I} n(\widehat{X})$ can be identified with the complexification of $\mathcal{L}(X) / \mathcal{I} n(X)$; in particular, these two quotient algebras have the same dimension. Our main tools are the notion of complexification of a real Banach space, the Wedderburn representation theorem for semisimple algebras and some techniques of Fredholm theory. In [11] we obtained similar results in the case when $X$ is a complex Banach space. In that case the results and proofs were simpler; in particular, $\mathcal{L}\left(Y_{i j}\right) / \mathcal{I} n\left(Y_{i j}\right)$ was always isomorphic to $\mathbb{C}$.

Along the paper $X, Y, Z, \ldots$ will be real or complex Banach spaces, $X^{*}$ the dual space of $X$, and $\mathcal{L}(X, Y)$ the (continuous linear) operators from $X$ into $Y$. We will write $\mathcal{L}(X)$ instead of $\mathcal{L}(X, X)$ and $I$ will denote the identity map on a Banach space. An operator $T \in \mathcal{L}(X, Y)$ is upper semi-Fredholm if its range $R(T)$ is closed and its kernel $N(T)$ is finite-dimensional; lower semiFredholm if its range $R(T)$ is closed and finite-codimensional; and Fredholm if it is upper and lower semi-Fredholm. We denote these classes of operators by $\Phi_{+}, \Phi_{-}$and $\Phi$, respectively.

Given a closed subspace $M$ of a Banach space $X$ we denote by $J_{M}$ the natural injection of $M$ into $X$ and by $Q_{M}$ the quotient map of $X$ onto $X / M$. An operator $T \in \mathcal{L}(X, Y)$ is strictly singular if there is no infinite-dimensional subspace $M$ of $X$ such that the restriction $T J_{M}$ is an isomorphism; strictly cosingular if there is no infinite-codimensional closed 
subspace $N$ of $Y$ such that $Q_{N} T$ is surjective; and inessential if for every $S \in \mathcal{L}(Y, X)$, the operator $I-S T$ is Fredholm. We denote these classes of operators by $\mathcal{S S}, \mathcal{S C}$ and $\mathcal{I} n$, respectively. Moreover, $\mathcal{K}$ denotes the ideal of compact operators. Note that both $\mathcal{S S}$ and $\mathcal{S C}$ are properly contained in $\mathcal{I} n$.

We say that a closed two-sided ideal $\mathcal{A}(X)$ in $\mathcal{L}(X)$ is a Fredholm ideal when an operator $T \in \mathcal{L}(X)$ is Fredholm if and only if the corresponding class is invertible in $\mathcal{L}(X) / \mathcal{A}(X)$. It follows from well-known results in Fredholm theory that $\mathcal{A}(X)$ is a Fredholm ideal if and only if $\mathcal{A}(X)$ contains the ideal of finite range operators and is contained in the ideal of inessential operators. As examples, we have $\mathcal{K}(X), \mathcal{S S}(X), \mathcal{S C}(X), \mathcal{I} n(X)$, and the perturbation classes of the semi-Fredholm operators $P \Phi_{+}(X)$ and $P \Phi_{-}(X)$. We refer to $[9,10]$ for the properties of these Fredholm ideals.

2. Preliminaries. Here we collect some basic results relating a Banach space to its complexification, and operators between two real Banach spaces with operators between their complexifications. Along this section, $X$ and $Y$ will be real Banach spaces.

Recall that the complexification $\widehat{X}$ of a real Banach space $X$ (see, for example, [16, p. 81]) is defined as the space

$$
\widehat{X}=\{x+i y: x, y \in X\} \equiv X \times X
$$

with the external operation given by

$$
(a+i b)(x+i y):=a x-b y+i(a y+b x) \equiv(a x-b y, a y+b x), \quad a, b \in \mathbb{R},
$$

and the norm defined by

$\|x+i y\|:=\max \left\{\left(\|a x-b y\|^{2}+\|a y+b x\|^{2}\right)^{1 / 2}: a, b \in \mathbb{R}, a^{2}+b^{2}=1\right\}$.

Set $\|(x, y)\|_{2}=\left(\|x\|^{2}+\|y\|^{2}\right)^{1 / 2}$ for $x, y \in X$. It is easy to check that

$$
\|(x, y)\|_{2} \leq\|x+i y\| \leq \sqrt{2}\|(x, y)\|_{2} \text {. }
$$

Similarly, if $A$ is a real Banach algebra, we can define the complexification $\widehat{A}$ of $A$, where the product in $\widehat{A}$ is given by

$$
(u+i v)(x+i y):=u x-v y+i(u y+v x) \text {. }
$$

We refer to [3, Section 13] for additional information.

The following result is a direct consequence of (1) and the definition of the map $U_{X}$.

Proposition 2.1. The map $U_{X}: \widehat{X} \rightarrow X \times X$ defined by $U_{X}(x+i y):=$ $(x, y)$ has the following properties:

(i) $U_{X}$ and $U_{X}^{-1}$ are continuous: $z_{n} \rightarrow z$ if and only if $U_{X} z_{n} \rightarrow U_{X} z$.

(ii) $U_{X}$ is bijective and additive. 
(iii) $M$ is a subspace of $\widehat{X} \Rightarrow U_{X}(M)$ is a subspace of $X \times X$.

(iv) Let $N$ be a subspace of $X \times X$. Then

$$
U_{X}^{-1}(N) \text { is a subspace of } \widehat{X} \Leftrightarrow(-y, x) \in N \text { for each }(x, y) \in N \text {. }
$$

Let $A, B \in \mathcal{L}(X, Y)$. Then

$$
(A+i B)(x+i y):=A x-B y+i(A y+B x)
$$

defines an operator $A+i B \in \mathcal{L}(\widehat{X}, \widehat{Y})$ that satisfies

$$
\max \{\|A\|,\|B\|\} \leq\|A+i B\| \leq \sqrt{2}(\|A\|+\|B\|) .
$$

Conversely, given $T \in \mathcal{L}(\widehat{X}, \widehat{Y})$, if we put $T(x+i 0):=A x+i B x$, then we obtain $A, B \in \mathcal{L}(X, Y)$ such that $T=A+i B$.

As a consequence of the previous arguments we get the following result.

Proposition 2.2. We can identify the space $\mathcal{L}(\widehat{X}, \widehat{Y})$ and the complexification $\widehat{\mathcal{L}(X, Y)}$ of $\mathcal{L}(X, Y)$. In the case $X=Y$, this identification is an isomorphism between the algebras $\mathcal{L}(\widehat{X})$ and $\widehat{\mathcal{L}(X)}$.

Let $U_{X}: \widehat{X} \rightarrow X \times X$ and $U_{Y}: \widehat{Y} \rightarrow Y \times Y$ be the maps defined in Proposition 2.1. We have the commutative diagram

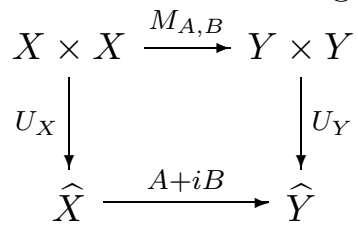

where $M_{A, B}$ denotes the operator defined by the matrix $\left(\begin{array}{cc}A & -B \\ B & A\end{array}\right)$.

The following result is a direct consequence of the definition of operator ideal in the sense of Pietsch [19].

Proposition 2.3. Let $\mathcal{A}$ be an operator ideal. Then $A, B \in \mathcal{A}(X, Y)$ if and only if $M_{A, B} \in \mathcal{A}(X \times X, Y \times Y)$.

However, the relation between the properties of $A, B$ and the properties of $A+i B$ is not so direct. One reason for this is that the concept of operator ideal does not mix real spaces and complex spaces. Moreover, even in the case in which the definition of the operator ideal makes sense both in the real and in the complex case, there are some difficulties because the relation between $X \times X$ and $\widehat{X}$ is not $\mathbb{C}$-linear.

Proposition 2.4. Let $A, B \in \mathcal{L}(X, Y)$. Then $A+i B \in \mathcal{K}(\widehat{X}, \widehat{Y})$ if and only if $A, B \in \mathcal{K}(X, Y)$.

Proof. Let $A, B \in \mathcal{K}$ and $\left(z_{n}\right) \subset \widehat{X}$ bounded. If $z_{n}=x_{n}+i y_{n}$, then $(A+i B)\left(z_{n}\right)=A x_{n}-B y_{n}+i\left(A y_{n}+B x_{n}\right)$. Since $\left(x_{n}\right)$ and $\left(y_{n}\right)$ are bounded, passing to subsequences we get $\left(A x_{n_{k}}\right),\left(B y_{n_{k}}\right),\left(B x_{n_{k}}\right),\left(A y_{n_{k}}\right)$ convergent. Hence $(A+i B)\left(z_{n_{k}}\right)$ converges and $A+i B \in \mathcal{K}$. 
Conversely, let $A+i B \in \mathcal{K}$ and $\left(x_{n}\right) \subset X$ bounded. Then $\left(x_{n}+i 0\right) \subset \widehat{X}$ is bounded and there is a convergent subsequence $(A+i B)\left(x_{n_{k}}\right)=\left(A x_{n_{k}}+\right.$ $\left.i B x_{n_{k}}\right)$. Thus $\left(A x_{n_{k}}\right),\left(B x_{n_{k}}\right)$ are convergent and $A, B \in \mathcal{K}$.

Let $A+i B \in \mathcal{L}(\widehat{X}, \widehat{Y})$. We have seen that there exist two homeomorphisms $U_{X}: \widehat{X} \rightarrow X \times X$ and $U_{Y}: \widehat{Y} \rightarrow Y \times Y$ so that $A+i B=U_{Y} M_{A, B} U_{X}^{-1}$.

Proposition 2.5. Let $\mathcal{A}$ be one of the classes of Fredholm $\Phi$, upper semi-Fredholm $\Phi_{+}$or lower semi-Fredholm operators $\Phi_{-}$. Let $A, B \in \mathcal{L}(X, Y)$. Then $A+i B \in \mathcal{A}(\widehat{X}, \widehat{Y})$ if and only if $M_{A, B} \in \mathcal{A}(X \times X, Y \times Y)$.

Proof. It is enough to observe that

(a) $R\left(M_{A, B}\right)$ is closed if and only if $R(A+i B)$ is closed,

(b) $\operatorname{dim}_{\mathbb{R}} N\left(M_{A, B}\right)=2 \operatorname{dim}_{\mathbb{C}} N(A+i B)$,

(c) $\operatorname{codim}_{\mathbb{R}} R\left(M_{A, B}\right)=2 \operatorname{codim}_{\mathbb{C}} R(A+i B)$.

Proposition 2.6. Let $\mathcal{A}$ be one of the Fredholm ideals $\mathcal{S} \mathcal{S}, \mathcal{S C}, \mathcal{I} n, P \Phi_{+}$ or $P \Phi_{-}$. If $A, B \in \mathcal{A}(X, Y)$ then $A+i B \in \mathcal{A}(\widehat{X}, \widehat{Y})$.

Proof. The result for $\mathcal{S S}$ and $\mathcal{S C}$ follows easily from the identification $A+i B=U_{Y} M_{A, B} U_{X}^{-1}$ and the properties of $U_{Y}$ and $U_{X}$ given in Proposition 2.1 .

The result for the perturbation classes $\mathcal{I}_{n}=P \Phi, P \Phi_{+}$and $P \Phi_{-}$follows from Proposition 2.5.

REMARK 2.7. It is not known whether $A+i B \in \mathcal{S S}(\widehat{X}, \widehat{Y})$ implies $A, B \in \mathcal{S S}(X, Y)$. Note that $X \times X$ has more subspaces than $\widehat{X}$. So it is reasonable to expect a negative answer.

Proposition 2.8. Let $\mathcal{U}$ be an operator ideal. Suppose that $K, L \in$ $\mathcal{U}(X, Y)$ implies $K+i L \in \mathcal{U}(\widehat{X}, \widehat{Y})$. Then

$$
\operatorname{dim}_{\mathbb{C}} \mathcal{L}(\widehat{X}, \widehat{Y}) / \mathcal{U}(\widehat{X}, \widehat{Y}) \leq \operatorname{dim}_{\mathbb{R}} \mathcal{L}(X, Y) / \mathcal{U}(X, Y) .
$$

Proof. Suppose $\operatorname{dim} \mathcal{L}(X, Y) / \mathcal{U}(X, Y)=n$. Then there exist $A_{1}, \ldots, A_{n}$ $\in \mathcal{L}(X, Y)$ such that, for every $T=A+i B \in \mathcal{L}(\widehat{X}, \widehat{Y})$ we can find real numbers $\lambda_{1}, \ldots, \lambda_{n}$ and $\mu_{1}, \ldots, \mu_{n}$, and $K, L \in \mathcal{U}(X, Y)$, such that $A=$ $\lambda_{1} A_{1}+\cdots+\lambda_{n} A_{n}+K$ and $B=\mu_{1} A_{1}+\cdots+\mu_{n} A_{n}+L$. Thus

$$
T=\left(\lambda_{1}+i \mu_{1}\right)\left(A_{1}+i 0\right)+\cdots+\left(\lambda_{n}+i \mu_{n}\right)\left(A_{n}+i 0\right)+(K+i L)
$$

with $K+i L \in \mathcal{U}(\widehat{X}, \widehat{Y})$.

Remark 2.9. The condition " $K, L \in \mathcal{U}(X, Y)$ implies $K+i L \in \mathcal{U}(\widehat{X}, \widehat{Y})$ " is equivalent to the inclusion $\widehat{\mathcal{U}(X, Y)} \subseteq \mathcal{U}(\widehat{X}, \widehat{Y})$. In the case $X=Y$ we get a natural surjection from $\mathcal{L}(\widehat{X) / \mathcal{U}}(X) \simeq \widehat{\mathcal{L}(X) / \widehat{\mathcal{U}(X)}}$ onto $\mathcal{L}(\widehat{X}) / \mathcal{U}(\widehat{X})$. Thus, if $\operatorname{dim}_{\mathbb{R}} \mathcal{L}(X) / \mathcal{U}(X)=\operatorname{dim}_{\mathbb{C}} \mathcal{L}(\widehat{X}) / \mathcal{U}(\widehat{X})$, we can identify $\mathcal{L}(\widehat{X}) / \mathcal{U}(\widehat{X})$ and the complexification $\mathcal{L}(\widehat{X) / \mathcal{U}}(X)$. 
3. Main results. We say that a Banach space $X$ is indecomposable if it cannot be decomposed as the direct sum of two infinite-dimensional closed subspaces; equivalently, if for each projection $P \in \mathcal{L}(X)$, the kernel or the range of $P$ is finite-dimensional.

In this section, for a real Banach space $X$ such that $\mathcal{L}(X) / \mathcal{I} n(X)$ is finite-dimensional, we obtain a decomposition of $X$ into indecomposable complemented subspaces $Z_{j}$ such that each of the algebras $\mathcal{L}\left(Z_{j}\right) / \mathcal{I} n\left(Z_{j}\right)$ is isomorphic to one of the real division algebras $\mathbb{R}, \mathbb{C}$ or $\mathbb{H}$. Moreover, we show that $\mathcal{L}(X) / \mathcal{I} n(X)$ and $\mathcal{L}(\widehat{X}) / \mathcal{I} n(\widehat{X})$ have the same dimension, and we relate the properties of the summands of the decompositions of $X$ and $\widehat{X}$.

The following result describes $\mathcal{L}(X) / \mathcal{I} n(X)$ when it is finite-dimensional. If $\mathbb{D}$ is a division algebra, we denote by $M_{n}(\mathbb{D})$ the algebra of all $n \times n$ matrices with entries in $\mathbb{D}$.

Theorem 3.1. Let $X$ be a real Banach space such that $\mathcal{L}(X) / \mathcal{I} n(X)$ is finite-dimensional. Then there exist positive integers $n_{1}, \ldots, n_{k}$ so that

$$
\mathcal{L}(X) / \mathcal{I} n(X) \equiv M_{n_{1}}\left(\mathbb{D}_{1}\right) \oplus \cdots \oplus M_{n_{k}}\left(\mathbb{D}_{k}\right),
$$

where each $\mathbb{D}_{i}$ is $\mathbb{R}, \mathbb{C}$ or $\mathbb{H}$.

Proof. It was proved by Kleinecke [14] that the inessential operators $\mathcal{I} n(X)$ can be characterized as the inverse image under the quotient map of the (Jacobson) radical of the algebra $\mathcal{L}(X) / \mathcal{K}(X)$. As a consequence, the quotient algebra $\mathcal{L}(X) / \mathcal{I} n(X)$ is semisimple, i.e., its radical is $\{0\}[3$, Definition 24.13]. Thus the result is a direct consequence of the Wedderburn structure theorem for semisimple finite-dimensional algebras [18, Section $3.5]$.

REMARK 3.2. In the complex case Theorem 3.1 is valid, with $\mathbb{D}_{i}=\mathbb{C}$ for all $i=1, \ldots, k$. See $[11]$.

Remark 3.3. Let $X$ be a real or complex space. If $\mathcal{U}$ is a Fredholm ideal and $L(X) / \mathcal{U}(X)$ is semisimple, then $\mathcal{U}(X)=\mathcal{I} n(X)$. This is true, in particular, for the real spaces $X$ for which $L(X) / \mathcal{S S}(X)$ is $\mathbb{R}, \mathbb{C}$ or $\mathbb{H}$. See, for example, [1], [2], [7], [12], and Koszmider's real $C(K)$ space for which $L(C(K)) / \mathcal{S} \mathcal{S}(C(K)) \equiv C(K)[15]$.

Let us see that each algebra $M_{n_{1}}\left(\mathbb{D}_{1}\right) \oplus \cdots \oplus M_{n_{k}}\left(\mathbb{D}_{k}\right)$ is isomorphic to $\mathcal{L}(X) / \mathcal{I} n(X)$ for some real Banach space $X$.

ExAmples 3.4. In [7] Ferenczi constructed a real Banach space $X_{\mathbb{H}}$ such that $\mathcal{L}\left(X_{\mathbb{H}}\right) / \mathcal{S} \mathcal{S}\left(X_{\mathbb{H}}\right) \equiv \mathbb{H}$. This space admits a Schauder decomposition $X=\bigoplus_{k=1}^{\infty} M_{k}$ with $\operatorname{dim} M_{k}=4$ for all $k$. For each $n$, we can define $n$ subspaces $Y_{i}:=\bigoplus_{k=1}^{\infty} M_{2^{i} k}, i=1, \ldots, n$. Moreover, we set $Y_{0}=X_{\mathbb{H}}$.

Clearly $Y_{i} \subset Y_{i-1}$ with $\operatorname{dim} Y_{i-1} / Y_{i}=\infty$, and it is easy to check as in [7] that $\mathcal{L}\left(Y_{i}\right) / \mathcal{S S}\left(Y_{i}\right) \equiv \mathbb{H}$ for each $i=1, \ldots, n$. 
For each $i=1, \ldots, n, Y_{i}$ is hereditarily indecomposable. Therefore $\mathcal{L}\left(Y_{i-1}, Y_{i}\right)=\mathcal{S S}\left(Y_{i-1}, Y_{i}\right)$; hence (see Remark 3.9 below)

$$
\mathcal{L}\left(Y_{i-1}, Y_{i}\right)=\mathcal{I} n\left(Y_{i-1}, Y_{i}\right) \text { and } \mathcal{L}\left(Y_{i}, Y_{i-1}\right)=\mathcal{I} n\left(Y_{i}, Y_{i-1}\right) .
$$

Let us denote by $Y_{i}^{n_{i}}$ the product of $n_{i}$ copies of $Y_{i}$. Clearly, if we take $X=Y_{1}^{n_{1}} \oplus \cdots \oplus Y_{k}^{n_{k}}$, then

$$
\mathcal{L}(X) / \mathcal{S} \mathcal{S}(X) \equiv M_{n_{1}}(\mathbb{H}) \oplus \cdots \oplus M_{n_{k}}(\mathbb{H}) .
$$

Analogously, the real Banach space $X_{\mathrm{GM}}$ constructed in [12] such that $\mathcal{L}\left(X_{\mathrm{GM}}\right) / \mathcal{S S}\left(X_{\mathrm{GM}}\right) \equiv \mathbb{R}$ is hereditarily indecomposable and has a Schauder basis, and the real Banach space $X_{\mathbb{C}}$ in $[7]$ such that $\mathcal{L}\left(X_{\mathbb{C}}\right) / \mathcal{S S}\left(X_{\mathbb{C}}\right) \equiv \mathbb{C}$ is hereditarily indecomposable and admits a Schauder decomposition into subspaces of dimension 2. So we can construct subspaces $Y_{i}(i=1, \ldots, n)$ of $X_{\mathrm{GM}}$ (respectively $X_{\mathbb{C}}$ ) satisfying $(2)$ and (3) with $\mathbb{R}$ (respectively $\mathbb{C}$ ) instead of $\mathbb{H}$.

Using a suitable combination of subspaces $Y_{i}$ of the previously mentioned three kinds, we can obtain a real Banach space $X$ which can be written as the direct sum of $n_{1}+\cdots+n_{k}$ indecomposable subspaces such that $\mathcal{L}(X) / \mathcal{S} \mathcal{S}(X) \equiv \mathcal{L}(X) / \mathcal{I} n(X)$ is isomorphic to $M_{n_{1}}\left(\mathbb{D}_{1}\right) \oplus \cdots \oplus M_{n_{k}}\left(\mathbb{D}_{k}\right)$, where each $\mathbb{D}_{i}$ is $\mathbb{R}, \mathbb{C}$ or $\mathbb{H}$.

Let $\mathcal{U}(X)$ be a Fredholm ideal, let $\pi: \mathcal{L}(X) \rightarrow \mathcal{L}(X) / \mathcal{U}(X)$ denote the quotient map, and let $e$ denote the identity of $\mathcal{L}(X) / \mathcal{U}(X)$.

The following result will allow us to lift idempotents in $\mathcal{L}(X) / \mathcal{U}(X)$ to projections in $\mathcal{L}(X)$.

Proposition 3.5. Let $X$ be a Banach space and let $\mathcal{U}(X)$ be a Fredholm ideal in $\mathcal{L}(X)$. Let $p_{1}, \ldots, p_{n} \in \mathcal{L}(X) / \mathcal{U}(X)$ be nonzero idempotents such that $p_{i} p_{j}=0$ for $i \neq j$ and $p_{1}+\cdots+p_{n}=e$. Then there exist projections $P_{1}, \ldots, P_{n} \in \mathcal{L}(X)$ such that $P_{i} P_{j}=0$ for $i \neq j, P_{1}+\cdots+P_{n}=I$ and $p_{i}=\pi\left(P_{i}\right)$ for each $i$.

Proof. We define $x:=p_{1}+2 p_{2}+\cdots+n p_{n} \in \mathcal{L}(X) / \mathcal{U}(X)$.

First we suppose that $X$ is a complex space. Observe that the spectrum of $x$ is the set $\{1, \ldots, n\}$. Indeed, for every $\lambda \in \mathbb{C}$,

$$
\lambda e-x=\lambda\left(p_{1}+\cdots+p_{n}\right)-\left(p_{1}+2 p_{2}+\cdots+n p_{n}\right)=\sum_{k=1}^{n}(\lambda-k) p_{k} ;
$$

hence $(\lambda e-x)^{-1}=\sum_{k=1}^{n}(\lambda-k)^{-1} p_{k}$ for $\lambda \notin\{1, \ldots, n\}$.

We choose $T \in \mathcal{L}(X)$ such that $\pi(T)=x$; hence the only possible accumulation points of $\sigma(T)$ are $1, \ldots, n$. So we can take $n$ disjoint, simple closed curves $\Gamma_{k}(k=1, \ldots, n)$ in $\mathbb{C} \backslash \sigma(T)$ such that $k$ is enclosed by $\Gamma_{k}$, $l$ is not enclosed by $\Gamma_{k}$ for $l \in\{1, \ldots, n\}$ with $k \neq l$, and every point in $\sigma(T)$ is enclosed by one of the curves $\Gamma_{k}$. It follows from the properties of 
the analytic functional calculus [20] that the expressions

$$
P_{k}:=\frac{1}{2 \pi i} \int_{\Gamma_{k}}(\lambda I-T)^{-1} d \lambda \quad(k=1, \ldots, n)
$$

define projections that satisfy $P_{i} P_{j}=0$ for $i \neq j$ and $P_{1}+\cdots+P_{n}=I$. Moreover, by the continuity of $\pi$, for each $k=1, \ldots, n$,

$$
\pi\left(P_{k}\right)=\frac{1}{2 \pi i} \int_{\Gamma_{k}}(\lambda e-\pi(T))^{-1} d \lambda=\frac{1}{2 \pi i} \int_{\Gamma_{k}}(\lambda-k)^{-1} p_{k} d \lambda=p_{k} .
$$

In the case that $X$ is a real space, we take $T \in \mathcal{L}(X)$ as in the complex case, and consider the complexification $\widehat{X}$ of $X$, the operator $\widehat{T}:=T+i 0 \in$ $\mathcal{L}(\widehat{X})$ and the Fredholm ideal

$$
\mathcal{U}(\widehat{X}):=\{A+i B: A, B \in \mathcal{U}(X)\} .
$$

So we can identify $\mathcal{L}(\widehat{X}) / \mathcal{U}(\widehat{X})$ with the complexification of $\mathcal{L}(X) / \mathcal{U}(X)$. It is easy to check that $\lambda I-\widehat{T}$ is invertible if and only if so is $\bar{\lambda} I-\widehat{T}$, with

$$
(\bar{\lambda} I-\widehat{T})^{-1}=\overline{(\lambda I-\widehat{T})^{-1}} .
$$

So $\sigma(\widehat{T})$ is symmetric with respect to the real axis, and we can proceed as in the complex case taking the curves $\Gamma_{k}$ symmetric with respect to the real axis. We denote by $\Gamma_{k}^{+}$and $\Gamma_{k}^{-}$the intersections of $\Gamma_{k}$ with the upper and lower complex half-planes respectively. Then

$$
\begin{aligned}
\int_{\Gamma_{k}}(\lambda I-\widehat{T})^{-1} d \lambda & =\int_{\Gamma_{k}^{+}}(\lambda I-\widehat{T})^{-1} d \lambda+\int_{\Gamma_{k}^{-}}(\lambda I-\widehat{T})^{-1} d \lambda \\
& =\int_{\Gamma_{k}^{+}}(\lambda I-\widehat{T})^{-1} d \lambda-\int_{\Gamma_{k}^{+}}(\bar{\lambda} I-\widehat{T})^{-1} d \bar{\lambda} \\
& =2 i \operatorname{Im} \int_{\Gamma_{k}^{+}}(\lambda I-\widehat{T})^{-1} d \lambda .
\end{aligned}
$$

Thus the imaginary part of the projection $\widehat{P}_{k}$ obtained from $\widehat{T}$ is 0 , hence $\widehat{P}_{k}=P_{k}+i 0$ for some projection $P_{k} \in \mathcal{L}(X)$, and clearly $\pi\left(P_{k}\right)=p_{k}$.

Recall that Frobenius proved that the only associative, finite-dimensional division algebras over the real field are the real numbers $\mathbb{R}$, the complex numbers $\mathbb{C}$, and the quaternion numbers $\mathbb{H}$ (see [13, Corollary IX.6.8]).

TheOREM 3.6. Let $X$ be a real Banach space such that $\mathcal{L}(X) / \mathcal{I} n(X)$ is finite-dimensional. Then $X$ is indecomposable if and only if $\mathcal{L}(X) / \mathcal{I} n(X)$ is a division algebra, i.e., if and only if it is isomorphic to $\mathbb{R}, \mathbb{C}$ or $\mathbb{H}$.

Proof. If $\mathcal{L}(X) / \mathcal{I} n(X)$ is a division algebra, then 0 and the identity are the only idempotents it contains. Hence, for each projection in $\mathcal{L}(X)$ either the kernel or the range is finite-dimensional. Thus, $X$ is indecomposable. 
Conversely, suppose that $\mathcal{L}(X) / \mathcal{I} n(X)$ is not a division algebra. Then by Theorem 3.1, there exists a nontrivial idempotent $q \in \mathcal{L}(X) / \mathcal{I} n(X)$. By Proposition 3.5 applied to $q$ and $e-q$, we can lift $q$ to a projection $P \in \mathcal{L}(X)$, and clearly both $N(P)$ and $R(P)$ are infinite-dimensional. Thus, $X$ is not indecomposable.

REMARK 3.7. In the complex case Theorem 3.6 is also valid, but in this case $\mathbb{C}$ is the only division algebra. See [11].

The following concepts were introduced in [8] and [11].

Definition 3.8. We say that two Banach spaces $X$ and $Y$ are essentially incomparable if $\mathcal{L}(X, Y)=\mathcal{I} n(X, Y)$.

We say that $X$ and $Y$ are essentially isomorphic if $\Phi(X, Y) \neq \emptyset$.

REMARK 3.9. The relations of being essentially incomparable and being essentially isomorphic are symmetric [8], i.e., $\mathcal{L}(X, Y)=\mathcal{I} n(X, Y)$ if and only if $\mathcal{L}(Y, X)=\mathcal{I} n(Y, X)$, and $\Phi(X, Y) \neq \emptyset$ if and only if $\Phi(Y, X) \neq \emptyset$. Moreover, $X$ and $Y$ essentially isomorphic means that there exists a finitedimensional space $N$ such that $X$ is isomorphic to $Y \oplus N$ or $Y$ is isomorphic to $X \oplus N$.

The following result shows that, under some conditions, two indecomposable Banach spaces are either essentially incomparable or essentially isomorphic.

THEOREM 3.10. Let $X$ and $Y$ be indecomposable Banach spaces. Suppose that $\operatorname{dim} \mathcal{L}(X) / \mathcal{I} n(X)<\infty$ or $\operatorname{dim} \mathcal{L}(Y) / \mathcal{I} n(Y)<\infty$. Then either $\mathcal{L}(X, Y)=\mathcal{I} n(X, Y)$ or $\Phi(X, Y) \neq \emptyset$.

Proof. Suppose that $\operatorname{dim} \mathcal{L}(X) / \mathcal{I} n(X)<\infty$. Let $\pi: \mathcal{L}(X) \rightarrow \mathcal{L}(X) / \mathcal{I} n(X)$ denote the quotient map, and assume that $\mathcal{L}(X, Y) \neq \mathcal{I} n(X, Y)$.

Taking $T \in \mathcal{L}(X, Y) \backslash \mathcal{I} n(X, Y)$, we can find $S \in \mathcal{L}(Y, X)$ such that $I_{X}-S T$ is not a Fredholm operator; hence $\pi\left(I_{X}-S T\right)$ is not invertible. Since, by Theorem 3.6 and Remark 3.7, $\mathcal{L}(X) / \mathcal{I} n(X)$ is a division algebra, $\pi\left(I_{X}-S T\right)=0$. Then $S T$ is a Fredholm operator, hence $T$ is left invertible modulo $\mathcal{K}(X)$; equivalently, $T$ is an upper semi-Fredholm operator with complemented range [4, 4.3.2 Theorem]. Since $Y$ is indecomposable, $R(T)$ is finite-codimensional; hence $T \in \Phi(X, Y)$.

The proof of the case $\operatorname{dim} \mathcal{L}(Y) / \mathcal{I} n(Y)<\infty$ is similar.

Now we can give a real version of Proposition 2.9 in [11]. We denote by $\Phi_{l}$ (respectively, $\left.\Phi_{r}\right)$ the operators in $\Phi_{+}$(respectively, $\Phi_{-}$) with complemented range and kernel.

Proposition 3.11. For an infinite-dimensional Banach space $X$, the following assertions are equivalent: 
(i) $\mathcal{L}(X) / \mathcal{I} n(X)$ is a division algebra;

(ii) $\mathcal{L}(X)=\Phi(X) \cup \mathcal{I} n(X)$;

(iii) $\mathcal{L}(X, Y)=\Phi_{l}(X, Y) \cup \mathcal{I} n(X, Y)$ for every Banach space $Y$;

(iv) $\mathcal{L}(Z, X)=\Phi_{r}(Z, X) \cup \mathcal{I} n(Z, X)$ for every Banach space $Z$.

Proof. It is similar to the proof of Theorem 3.10.

Next we apply Theorem 3.1 to obtain a decomposition of real Banach spaces $X$ for which $\operatorname{dim} \mathcal{L}(X) / \mathcal{I} n(X)<\infty$ into indecomposable complemented subspaces.

THEOREm 3.12. Let $X$ be a real Banach space such that $\operatorname{dim} \mathcal{L}(X) / \mathcal{I} n(X)$ is finite-dimensional. Then there exist positive integers $n_{1}, \ldots, n_{k}$ and complemented subspaces $Y_{i j}$ of $X, i=1, \ldots, k, j=1, \ldots, n_{i}$, so that

(i) $X=\bigoplus_{i=1}^{k}\left(\bigoplus_{j=1}^{n_{i}} Y_{i j}\right)$;

(ii) for each $1 \leq i, i^{\prime} \leq k, 1 \leq j \leq n_{i}$ and $1 \leq j^{\prime} \leq n_{i^{\prime}}$,

$$
\mathcal{L}\left(Y_{i j}, Y_{i^{\prime} j^{\prime}}\right) / \mathcal{I} n\left(Y_{i j}, Y_{i^{\prime} j^{\prime}}\right) \equiv \begin{cases}\mathbb{D}_{i} & \text { for } i=i^{\prime}, \\ \{0\} & \text { for } i \neq i^{\prime} .\end{cases}
$$

In particular, each $Y_{i j}$ is indecomposable; $Y_{i j}$ and $Y_{i^{\prime} j^{\prime}}$ are essentially isomorphic for $i=i^{\prime}$, and essentially incomparable for $i \neq i^{\prime}$.

Proof. (i) By Theorem 3.1, $\mathcal{L}(X) / \mathcal{I} n(X)$ is isomorphic to an algebra

$$
M_{n_{1}}\left(\mathbb{D}_{1}\right) \oplus \cdots \oplus M_{n_{k}}\left(\mathbb{D}_{k}\right),
$$

where each $\mathbb{D}_{i}$ is $\mathbb{R}, \mathbb{C}$ or $\mathbb{H}$. So we can identify each $q \in \mathcal{L}(X) / \mathcal{I} n(X)$ with a $k$-tuple of square matrices.

For $1 \leq i \leq k$ and $1 \leq j \leq n_{i}$ we consider $p_{i j} \in \mathcal{L}(X) / \mathcal{I} n(X)$ such that all the matrices but the $i$ th in its associated $k$-tuple are 0 , and the $i$ th matrix has 1 in the $j$ th entry of its diagonal and 0 in the remaining entries.

Clearly the elements of $\left\{p_{i j}: 1 \leq i \leq k, 1 \leq j \leq n_{i}\right\}$ are idempotents, the product of two different ones is 0 , and $\sum_{i=1}^{k} \sum_{j=1}^{n_{i}} p_{i j}$ is the identity in $\mathcal{L}(X) / \mathcal{I} n(X)$. Applying Proposition 3.5 we obtain projections $\left\{P_{i j}: 1 \leq\right.$ $\left.i \leq k, 1 \leq j \leq n_{i}\right\}$ in $\mathcal{L}(X)$ such that the product of two different ones is 0 and $\sum_{i=1}^{k} \sum_{j=1}^{n_{i}} P_{i j}=I$. Thus

$$
X=\bigoplus_{i=1}^{k}\left(\bigoplus_{j=1}^{n_{i}} R\left(P_{i j}\right)\right)
$$

and taking $Y_{i j}:=R\left(P_{i j}\right)$, we obtain (i).

(ii) Consider the quotient map $\pi: \mathcal{L}(X) \rightarrow \mathcal{L}(X) / \mathcal{I} n(X)$. Note that the projections obtained in part (i) satisfy $\pi\left(P_{i j}\right)=p_{i j}$ for each $i, j$.

By part (i), each operator in $\mathcal{L}(X)$ can be identified with a square matrix whose entries are operators in $\mathcal{L}\left(Y_{i j}, Y_{i^{\prime} j^{\prime}}\right)$ with $1 \leq i, i^{\prime} \leq k, 1 \leq j \leq n_{i}$ and 
$1 \leq j^{\prime} \leq n_{i^{\prime}}$. Moreover, each operator in $\mathcal{L}\left(Y_{i j}, Y_{i^{\prime} j^{\prime}}\right)$ can be identified with an operator $T \in \mathcal{L}(X)$ satisfying $T=P_{i^{\prime} j^{\prime}} T P_{i j}$.

If $i \neq i^{\prime}$, then for all $T \in \mathcal{L}(X)$, we have $\pi\left(P_{i^{\prime} j^{\prime}} T P_{i j}\right)=p_{i^{\prime} j^{\prime}} \pi(T) p_{i j}=0$, hence $P_{i^{\prime} j^{\prime}} T P_{i j}$ is inessential. Thus $\mathcal{L}\left(Y_{i j}, Y_{i^{\prime} j^{\prime}}\right)=\mathcal{I} n\left(Y_{i j}, Y_{i^{\prime} j^{\prime}}\right)$.

If $i=i^{\prime}$, a similar argument shows that $\mathcal{L}\left(Y_{i j}, Y_{i j^{\prime}}\right) / \mathcal{I} n\left(Y_{i j}, Y_{i j^{\prime}}\right) \equiv \mathbb{D}_{i}$.

The remaining assertion is a consequence of the previous parts, Theorem 3.6 and Theorem 3.10 .

REMARK 3.13. The decomposition of a semisimple finite-dimensional algebra given by Wedderburn's theorem is unique, up to isomorphisms. So the same is true for the decomposition given in Theorem 3.12, up to essential isomorphisms (i.e., Fredholm operators).

REMARK 3.14. An alternative proof of Theorem 3.12 can be obtained as follows. If $\operatorname{dim} \mathcal{L}(X) / \mathcal{I} n(X)=N$, then any decomposition of $X$ as a direct sum of (infinite-dimensional) complemented subspaces has at most $N$ summands. So we can find a decomposition of $X$ as a direct sum of indecomposable complemented subspaces $Y_{i}$. By Theorem 3.10, we can divide these complemented subspaces into $k$ sets of $n_{i}$ elements each, in such a way that two of them are essentially incomparable if they belong to different subsets, and they are essentially isomorphic if they belong to the same subset. By Theorem 3.6, the algebras $\mathcal{L}\left(Y_{i}\right) / \mathcal{I} n\left(Y_{i}\right)$ are isomorphic to $\mathbb{R}, \mathbb{C}$ or $\mathbb{H}$; hence

$$
\mathcal{L}(X) / \mathcal{I} n(X) \equiv M_{n_{1}}\left(\mathbb{D}_{1}\right) \oplus \cdots \oplus M_{n_{k}}\left(\mathbb{D}_{k}\right),
$$

where each $\mathbb{D}_{i}$ is $\mathbb{R}, \mathbb{C}$ or $\mathbb{H}$.

Now we describe a decomposition for the complexification $\widehat{X}$ of a real Banach space $X$ for which $\mathcal{L}(X) / \mathcal{I} n(X)$ is finite-dimensional. Note that, by Proposition $2.8, \mathcal{L}(\widehat{X}) / \mathcal{I} n(\widehat{X})$ is also finite-dimensional. We will see that these dimensions are the same, and we will describe the relations between the respective decompositions of $X$ and $\widehat{X}$.

We begin with the case in which $\mathcal{L}(X) / \mathcal{I} n(X)$ is a division algebra.

Proposition 3.15. Let $X$ be a real Banach space. If $\mathcal{L}(X) / \mathcal{I} n(X)$ is isomorphic to $\mathbb{R}, \mathbb{C}$ or $\mathbb{H}$, then

$$
\operatorname{dim}_{\mathbb{C}} \mathcal{L}(\widehat{X}) / \mathcal{I} n(\widehat{X})=\operatorname{dim}_{\mathbb{R}} \mathcal{L}(X) / \mathcal{I} n(X) .
$$

Proof. By Proposition 2.8, $\operatorname{dim}_{\mathbb{C}} \mathcal{L}(\widehat{X}) / \mathcal{I} n(\widehat{X}) \leq \operatorname{dim}_{\mathbb{R}} \mathcal{L}(X) / \mathcal{I} n(X)$. So it remains to prove the reverse inequality. Let $\pi: \mathcal{L}(\widehat{X}) \rightarrow \mathcal{L}(\widehat{X}) / \mathcal{I} n(\widehat{X})$ denote the quotient map.

Case $\mathcal{L}(X) / \mathcal{I} n(X) \equiv \mathbb{R}$. Clearly $X$ is infinite-dimensional, and it is enough to observe that the identity of $\widehat{X}$ does not belong to $\mathcal{I} n(\widehat{X})$.

Case $\mathcal{L}(X) / \mathcal{I} n(X) \equiv \mathbb{C}$. We take $A \in \mathcal{L}(X)$ such that its image in $\mathcal{L}(X) / \mathcal{I} n(X)$ is $i$. Since the spectrum of $\pi(A+i 0)$ in $\mathcal{L}(\widehat{X}) / \mathcal{I} n(\widehat{X})$ is non- 
empty and symmetric with respect to the real axis, it coincides with $\{i,-i\}$. Thus $\pi(A+i 0)$ is not a multiple of the unit element.

Case $\mathcal{L}(X) / \mathcal{I} n(X) \equiv \mathbb{H}$. Let $I, U, V, W \in \mathcal{L}(X)$ be representatives of the quaternions $1, i, j, k \in \mathcal{L}(X) / \mathcal{I} n(X)$. We shall show that

$$
e:=\pi(I+i 0), \quad u:=\pi(U+i 0), \quad v:=\pi(V+i 0) \quad \text { and } \quad w:=\pi(W+i 0)
$$

are linearly independent in $\mathcal{L}(\widehat{X}) / \mathcal{I} n(\widehat{X})$.

Let $a, b, c, d \in \mathbb{C}$ be such that $a e+b u+c v+d w=0$. Multiplying by $u$ on the left and on the right we get

$$
a u-b e+c w-d v=0 \text { and } a u-b e-c w+d v=0 .
$$

Adding these two formulas we get $a u-b e=0$, hence $a=b=0$ by the argument of the case $\mathcal{L}(X) / \mathcal{I} n(X) \equiv \mathbb{C}$. Similarly, subtracting the formulas and multiplying the result by $w$ we get $c=d=0$.

The next result shows that the complexification $\widehat{X}$ of an indecomposable real Banach space $X$ with $\mathcal{L}(X) / \mathcal{I} n(X)$ finite-dimensional is sometimes indecomposable and sometimes not.

Proposition 3.16. Let $X$ be a real Banach space.

(i) If $\mathcal{L}(X) / \mathcal{I} n(X) \equiv \mathbb{R}$, then $\mathcal{L}(\widehat{X}) / \mathcal{I} n(\widehat{X}) \equiv \mathbb{C}$ and $\widehat{X}$ is indecomposable.

(ii) If $\mathcal{L}(X) / \mathcal{I} n(X) \equiv \mathbb{C}$, then $\mathcal{L}(\widehat{X}) / \mathcal{I} n(\widehat{X}) \equiv \mathbb{C}^{2}$ and we can write $\widehat{X}=Y_{1} \oplus Y_{2}$ with $Y_{1}$ and $Y_{2}$ essentially incomparable, indecomposable spaces.

(iii) If $\mathcal{L}(X) / \mathcal{I} n(X) \equiv \mathbb{H}$, then $\mathcal{L}(\widehat{X}) / \mathcal{I} n(\widehat{X}) \equiv M_{2}(\mathbb{C})$ and we can write $\widehat{X}=Z_{1} \oplus Z_{2}$ with $Z_{1}$ and $Z_{2}$ essentially isomorphic, indecomposable spaces.

Proof. Remark 2.9 and Proposition 3.15 imply that, if $\mathcal{L}(X) / \mathcal{I} n(X)$ is isomorphic to $\mathbb{R}, \mathbb{C}$ or $\mathbb{H}$, then $\mathcal{L}(\widehat{X}) / \mathcal{I} n(\widehat{X})$ can be identified with the respective complexification $\mathbb{C}, \mathbb{C}^{2}$ or $M_{2}(\mathbb{C})$. So the decomposition we give for $\widehat{X}$ follows from the complex version of Theorem 3.12. See [11, proof of Theorem 2.18].

Proposition 3.17. Let $X$ be a real Banach space.

(i) If $\mathcal{L}(X) / \mathcal{I} n(X) \equiv M_{n}(\mathbb{R})$, then $\mathcal{L}(\widehat{X}) / \mathcal{I} n(\widehat{X}) \equiv M_{n}(\mathbb{C})$.

(ii) If $\mathcal{L}(X) / \mathcal{I} n(X) \equiv M_{n}(\mathbb{C})$, then $\mathcal{L}(\widehat{X}) / \mathcal{I} n(\widehat{X}) \equiv M_{n}(\mathbb{C}) \times M_{n}(\mathbb{C})$.

(iii) If $\mathcal{L}(X) / \mathcal{I} n(X) \equiv M_{n}(\mathbb{H})$, then $\mathcal{L}(\widehat{X}) / \mathcal{I} n(\widehat{X}) \equiv M_{2 n}(\mathbb{C})$.

Proof. In the three cases we have a decomposition $X=Y_{1} \oplus \cdots \oplus Y_{n}$ into pairwise essentially isomorphic complemented subspaces, with each $\mathcal{L}\left(Y_{i}\right) / \mathcal{I} n\left(Y_{i}\right)$ isomorphic to $\mathbb{R}, \mathbb{C}$ and $\mathbb{H}$, respectively. 
It is an immediate consequence of the definition that the complexification $\widehat{X}$ is isomorphic to $\widehat{Y}_{1} \oplus \cdots \oplus \widehat{Y}_{n}$. So it is enough to apply Proposition 3.16 to each factor.

Our last result resumes some of the previous ones.

Proposition 3.18. Let $X$ be a real Banach space such that $\mathcal{L}(X) / \mathcal{I} n(X)$ is finite-dimensional. Then $\mathcal{L}(\widehat{X}) / \mathcal{I} n(\widehat{X}) \equiv \mathcal{L}(\widehat{X) / \mathcal{I} n}(X)$.

Proof. Given real Banach spaces $Y$ and $Z$, it is easy to see that $\mathcal{L}(Y, Z)=$ $\mathcal{I} n(Y, Z)$ if and only if $\mathcal{L}(\widehat{Y}, \widehat{Z})=\mathcal{I} n(\widehat{Y}, \widehat{Z})$. Moreover, if $X=\bigoplus_{i, j} X_{i j}$ is the decomposition of $X$ given in Theorem 3.12 , then $\widehat{X}=\bigoplus_{i, j} \widehat{X}_{i j}$. Thus the present result is a consequence of Proposition 3.17.

\section{References}

[1] S. A. Argyros and A. Manoussakis, An indecomposable and unconditionally saturated Banach space, Studia Math. 159 (2003), 1-32.

[2] S. A. Argyros and A. Tolias, Methods in the theory of hereditarily indecomposable Banach spaces, Mem. Amer. Math. Soc. 170 (2004), no. 806.

[3] F. F. Bonsall and J. Duncan, Complete Normed Algebras, Springer, New York, 1973.

[4] S. R. Caradus, W. E. Pfaffenberger and B. Yood, Calkin Algebras and Algebras of Operators on Banach Spaces, Dekker, New York, 1974.

[5] V. Ferenczi, Operators on subspaces of hereditarily indecomposable Banach spaces, Bull. London Math. Soc. 29 (1997), 338-344.

[6] - Hereditarily finitely decomposable spaces, Studia Math. 123 (1997), 135-149.

[7] - Uniqueness of complex structure and real hereditarily indecomposable Banach spaces, Adv. Math. 213 (2007), 462-488.

[8] M. González, On essentially incomparable Banach spaces, Math. Z. 215 (1994), 621-629.

[9] —, The perturbation classes problem in Fredholm theory, J. Funct. Anal. 200 (2003), $65-70$.

[10] —, Banach spaces with small Calkin algebras, in: Banach Center Publ. 75, Inst. Math., Polish Acad. Sci., 2007, 159-170.

[11] M. González and J. M. Herrera, Spaces on which the essential spectrum of all the operators is finite, J. Operator Theory 53 (2005), 303-314.

[12] W. T. Gowers and B. Maurey, The unconditional basic sequence problem, J. Amer. Math. Soc. 6 (1993), 851-874.

[13] T. W. Hungerford, Algebra, Grad. Texts in Math. 73, Springer, New York, 1974 (eighth printing, 1996).

[14] D. Kleinecke, Almost finite, compact and inessential operators, Proc. Amer. Math. Soc. 14 (1963), 863-868.

[15] P. Koszmider, Banach spaces of continuous functions with few operators, Math. Ann. 330 (2004), 151-183.

[16] J. Lindenstrauss and L. Tzafriri, Classical Banach Spaces I. Sequence Spaces, Springer, New York, 1977.

[17] B. Maurey, Banach spaces with few operators, in: Handbook of the Geometry of Banach Spaces, North-Holland, 2003, 1247-1297. 
[18] R. S. Pierce, Associative Algebras, Grad. Texts in Math. 88, Springer, New York, 1982.

[19] A. Pietsch, Operator Ideals, North-Holland, Amsterdam, 1980.

[20] A. E. Taylor and D. C. Lay, Introduction to Functional Analysis, Wiley, New York, 1980.

Departamento de Matemáticas

Universidad de Cantabria

E-39071 Santander, Spain

E-mail: gonzalem@unican.es

herreraj@unican.es

Received October 20, 2006

Revised version July 7, 2007 